\title{
Oscillator Strengths and Lifetimes for the $P$ XIII Spectrum
}

\author{
A. J. Mania, F. R. T. Luna \\ Universidade Estadual de Santa Cruz, DCET, Ilhéus, Brazil \\ Email:mania@uesc.br
}

Received 3 May 2016; accepted 17 July 2016; published 20 July 2016

Copyright (C) 2016 by authors and Scientific Research Publishing Inc.

This work is licensed under the Creative Commons Attribution International License (CC BY). http://creativecommons.org/licenses/by/4.0/

c) (†) Open Access

\begin{abstract}
The P XIII spectrum has been analyzed by several authors using different light sources. The semiempirical oscillator strengths $(g f)$ and the lifetimes presented in this work for all known P XIII spectral lines and energy levels were carried out in a multi-configuration Hartree-Fock relativistic (HFR) approach. In this calculation, the electrostatic parameters were optimized by a leastsquares procedure in order to improve the adjustment of theoretical to experimental energy levels. The method produces $g$ f-values that are in agreement with intensity observations and lifetime values closer to the experimental ones.
\end{abstract}

\section{Keywords}

P XIII Spectrum, Atomic Transitions, Energy Levels, Oscillator Strengths, Lifetimes

\section{Introduction}

The ground state configuration of twelve times ionized phosphorus, P XIII is $1 \mathrm{~s}^{2} 2 \mathrm{~s}$ with the term ${ }^{2} \mathrm{~S}_{1 / 2}$ being this spectrum a member of the Li-like isoelectronic sequence with a complete core plus a single-electron valence shell. The ionization limit for this ion is estimated as being 4,934,000 $\pm 600(611.74 \pm 0.07 \mathrm{eV})$. This spectrum was originally analyzed in 1948 by H. A. Robinson to find the energy levels. These studies have being used in the compilation of Atomic Energy Levels published by C. E. Moore [1], although a wavelength list has never been published. R. L. Kelly in Ref. [2] has included some of these lines as given by Moore in his first compilation. Later in 1970, Fawcett [3], using a theta-pinch as light source, identified wavelengths of transitions occurring in the interval $22-41 \AA$ and 278 - $594 \AA$. Fawcett et al. [4] using laser-produced plasma experiments confirmed the identity of many of their previous obtained lines classification. Goldsmith et al. [5] added, revised and extended the analyses performed by Robinson and Fawcett, classifying 13 lines in the range 23 - $39 \AA$ with the transitions 2s-np, 2p-3s, and 2p-nd. Kasyanov et al. [6] and Dere [7] realized measurements of the transition $1 s^{2} 2 p^{2} P_{3 / 2}$ to the 
ground level $1 \mathrm{~s}^{2} 2 \mathrm{~s}^{2} \mathrm{~S}_{1 / 2}$. Deschepper et al. [8] using a Doppler-tuned X-ray absorption technique realized measurements to the transition $1 \mathrm{~s}^{2} 2 \mathrm{~s}^{2} \mathrm{~S}_{1 / 2}-1 \mathrm{~s} 2 \mathrm{~s} 2 \mathrm{p}^{4} \mathrm{P}_{1 / 2}$ and ${ }^{4} \mathrm{P}_{3 / 2}$. Edlén [9], using series formulae, has given results for the $1 \mathrm{~s}^{2} \mathrm{nl}$ systems that are in agreement with the expected experimental errors. Wavelengths observed by Fawcett and Ridgeley [10] and Goldsmith et al. [5] in the region $102-111 \AA$, were used in the spectral analysis to evaluate the $4 p$ and $4 \mathrm{f}$ levels, being that the $4 \mathrm{~s}$ levels were evaluated from isoelectronic sequence values for $2 \mathrm{p}-4 \mathrm{~s}$ separations. Aglitskii et al. [11] and Boiko et al. [12], using laser-produced plasma, have contributed to the spectral features that have been observed in transitions of doublet term of the configurations $1 \mathrm{~s} 2 \mathrm{~s}^{2}, 1 \mathrm{~s} 2 \mathrm{p}^{2}, 1 \mathrm{~s} 2 \mathrm{~s} 2 \mathrm{p}$, $1 \mathrm{~s} 2 \mathrm{~s} 3 \mathrm{p}, 1 \mathrm{~s} 2 \mathrm{~s} 4 \mathrm{p}, 1 \mathrm{~s} 2 \mathrm{p} 3 \mathrm{p}, 1 \mathrm{~s} 2 \mathrm{p} 4 \mathrm{p}$, where the levels were derived from energy separations calculated by Vainshtein and Safronova [13] [14]. The wavelength tables given by these publications [15] [16], are lines arranged by spectra, being some lines of P XIII spectra which are not in other references. Martin et al. [17] compiles all the energy levels of all the phosphorus ions, revising the earlier version of Moore of all the published data so far, as well as the unpublished data of Robinson. Hayes and Fawcett [18] give a new original contribution by using the same spectrum obtained in theta-pinch experiment earlier. Kelly [19] reviews all P XIII line identifications in his compilation. Recently, Wang et al. [20]-[22] has published data of oscillator strengths for $1 \mathrm{~s}^{2} 2 \mathrm{~s}$ to $1 \mathrm{~s}^{2} \mathrm{np}$, and $1 \mathrm{~s}^{2} 2 \mathrm{p}$ to $1 \mathrm{~s}^{2}$ nd transitions of the Lithium isoelectronic sequence.

\section{Methodology}

Computations of wavelengths made with the aid of a Hartree-Fock Relativistic (HFR) computer program package and a program of least-square procedure as given by Cowan [23] to adjust the values of the energetic parameters, comparing the data and calculating its consistency with the identification of known energy levels. The adjustable parameters are to be determined empirically to give the best possible fitting between the calculated eigenvalues and the observed energy levels. The fitting process is carried out by a self-consistent procedure until the parameter values no longer change from one iteration cycle to the next. The main purpose is to reach a fitting to the experimental energy levels, which minimizes the uncertainties as much as possible, using the least-squares method for each parity in which the standard deviation is less than one percent of the energy range covered by the energy levels. The optimized electrostatic parameters substitute their corresponding theoretical values and they are used again to calculate energy matrices, the determination of the oscillator strengths and lifetimes values. All strong configuration interactions are to be included and HFR method is used to given a better accuracy [24]. It should also be noticed, that at higher levels, the $j-j$ notation is better and it should be used to estimate the percentages of compositions.

The oscillator strengths $f\left(\gamma, \gamma^{\prime}\right)$ is a physical quantity related to line intensity I and transition probability $W\left(\gamma, \gamma^{\prime}\right)$, by:

$$
W\left(\gamma, \gamma^{\prime}\right)=\frac{2 \omega^{2} e^{2}}{m c^{3}}\left|f\left(\gamma, \gamma^{\prime}\right)\right|,
$$

With $I \propto g W\left(\gamma, \gamma^{\prime}\right) \propto g\left|f\left(\gamma, \gamma^{\prime}\right)\right|=g f$, being $m$ the electron mass, $e$ their charge, $\gamma$ the initial quantum state, $\omega=\left(E(\gamma)-E\left(\gamma^{\prime}\right)\right) / \hbar, E(\gamma)$ the initial state energy and $g=(2 J+1)$ is the number of degenerate quantum states with angular momentum $J$. Quantities with primes refer to the final state. In the equation above, the weighted oscillator strength, $g f$, is

$$
g f=\frac{8 \pi^{2} m c a_{0}^{2} \sigma}{3 h} \mathbf{S}
$$

where $\sigma=\left|E(\gamma)-E\left(\gamma^{\prime}\right)\right| / h c, h$ is Planck's constant; $c$ is the light velocity; and $a_{0}$ is the Bohr radius. The electric dipole line strength is defined by:

$$
\mathbf{S}=\left|\left\langle\gamma J\left\|\mathbf{P}^{1}\right\| \gamma^{\prime} J^{\prime}\right\rangle\right|^{2}
$$

This quantity is a measure of the total strength of the spectral line, including all possible transitions between $m^{\prime} m$ and $J_{z}$ eigenstates. The tensor operator $\mathbf{P}^{1}$ (first order) in the reduced matrix element is the classical dipole moment for the atom in units of $-e a_{0}$. To obtain $g f$, we need to calculate $\mathbf{S}$ first, or its square root

$$
\mathbf{S}_{\gamma^{\prime}}^{1 / 2}=\sqrt{\mathbf{S}_{\gamma^{\prime}}}=\left\langle\gamma J\left\|\mathbf{P}^{1}\right\| \gamma^{\prime} J^{\prime}\right\rangle
$$


In a multiconfiguration calculation we have to expand the wavefunction $|\gamma J\rangle$ in terms of single configuration wavefunction, $|\beta J\rangle$, for both upper and lower levels:

$$
|\gamma J\rangle=\sum_{\beta} y_{\beta J}^{\gamma}|\beta J\rangle
$$

Therefore, we can have the multiconfigurational expression for $\mathbf{S}_{\gamma^{\prime}}^{1 / 2}$

$$
\mathbf{S}_{\gamma^{\prime}}^{1 / 2}=\sum_{\beta} \sum_{\beta^{\prime}} y_{\beta J}^{\gamma}\left\langle\beta J\left\|P^{1}\right\| \beta^{\prime} J^{\prime}\right\rangle y_{\beta^{\prime} J^{\prime}}^{\gamma^{\prime}}
$$

The probability per unit time of an atom in a specific state $\gamma J$ to make a spontaneous transition to any state with lower energy is

$$
P(\gamma J)=\sum A\left(\gamma J, \gamma^{\prime} J^{\prime}\right)
$$

where $A\left(\gamma J, \gamma^{\prime} J^{\prime}\right)$ is the Einstein spontaneous emission transition probability rate for a transition from the $\gamma J$ to the $\gamma^{\prime} J^{\prime}$ state. The sum is over all $\gamma^{\prime} J^{\prime}$ states with $E\left(\gamma^{\prime} J^{\prime}\right)<E(\gamma J)$. The Einstein probability rate is related to $g f$ through the following relation by:

$$
g A=\frac{8 \pi^{2} e^{2} \sigma^{2}}{m c} g f
$$

Since the natural lifetime $\tau(\gamma J)=\left(\sum A\left(\gamma J, \gamma^{\prime} J^{\prime}\right)\right)^{-1}$. The natural lifetime is applicable to an isolated atom.

The interaction with matter or radiation will reduce the lifetime of a state. The values for $g f$ and lifetime given in Table 1 and Table 2, respectively, were calculated according to these equations. In order to obtain better values for oscillator strengths, we calculated the reduced matrix elements $\mathbf{P}^{1}$ by using optimized values of energy parameters which were adjusted from a least-squares calculation. In this adjustment, the code tries to fit experimental energy values by varying the electrostatic parameters. This procedure improves $\sigma$ values used in Equation (2) and $y_{\beta,}^{\gamma}$ and $y_{\beta^{\prime} J^{\prime}}^{\gamma^{\prime}}$ values used in Equation (6).

Wavelength values in vacuum were converted to air by the relation [25], $\lambda_{\text {vac }}=n \lambda_{\text {air }}$, where the index of refraction of standard air (dry air containing $0.03 \mathrm{CO}_{2}$ by volume at normal pressure and $T=15^{\circ} \mathrm{C}$ ) is

$$
n=1.0+8342.13 \times 10^{-8}+\frac{2406030.0}{130.0 \times 10^{8}-\sigma^{2}}+\frac{15997.0}{38.9 \times 10^{8}-\sigma^{2}} .
$$

\section{Results and Discussion}

In our fitting process, the standard deviation reached for each parity as $12 \mathrm{~cm}^{-1}$ and $5 \mathrm{~cm}^{-1}$, for even and odd configurations, respectively, is satisfactory for the aims of this work. Values for $g f$ and lifetime given in Table 1 and Table 2, respectively, were calculated by the previously described method. Table 1 shows the results of the comparison between wavelength values as calculated by the method and the observed. In Table 2, we present lifetimes, energy levels and an estimation of their percentage composition. For the even-parity configurations we have the following picture: $1 \mathrm{~s}^{2} 5 \mathrm{~g}, 1 \mathrm{~s}^{2} 6 \mathrm{~g}, 1 \mathrm{~s}^{2} 6 \mathrm{~s}, 1 \mathrm{~s}^{2} 7 \mathrm{~s}, 1 \mathrm{~s}^{2} 8 \mathrm{~s}, 1 \mathrm{~s}^{2} 7 \mathrm{~d}, 1 \mathrm{~s}^{2} 8 \mathrm{~d}, 1 \mathrm{~s}^{2} \mathrm{~s}^{2}$, and the series $1 \mathrm{~s} 2 \mathrm{pnp}(3 \leq n \leq 4)$. For the odd-parity case we study the configuration $1 s^{2} 6 h$, and the series $1 \mathrm{~s}^{2} \mathrm{nf}(4 \leq n \leq 6), 1 \mathrm{~s}^{2} \mathrm{np}(5 \leq n \leq 8)$, 1 s 2 snp $(2 \leq n \leq 4)$. The interpretation of the configuration levels structure was made by least-squares fit of the observed levels and we propose the new values possible of the energy levels marked with asterisk $(*)$ in the table. The oscillator strengths and lifetimes for the lithium-like ions are of astrophysical interest for photo-ionization modelling of elemental abundances in cosmic objects since an extensive data source is not currently available. Transitions in this ion have been of particular importance in extrapolation analysis especially for the dense spectra from $N$-like sequence in which the phosphorus is one element in isoelectronic sequence linking lighter elements where the analysis is more extensive. Is also an important testing ground for the development of theoretical methods which attempt to calculate atomic structure of many-electron systems.

\section{Conclusion}

We have presented oscillator strengths and lifetimes for all known transitions in P XIII. The gf-values are better agreement with line intensity observations and lifetime values that are closer to the experimental ones. We have been stimulated by the need to determine both important parameters in the study of plasma laboratory and solar 
Table 1. Oscillator strengths and spectral lines for P XIII in the vacuum.

\begin{tabular}{|c|c|c|c|c|c|c|c|}
\hline \multirow[t]{2}{*}{$g f$-value } & \multirow[t]{2}{*}{ Int. } & \multicolumn{2}{|c|}{ Lambda $(\AA)$} & \multirow{2}{*}{$\begin{array}{c}\text { Levels }\left(\mathrm{cm}^{-1}\right) \\
\text { Even-Odd }\end{array}$} & \multirow[t]{2}{*}{ Configurations } & \multirow[t]{2}{*}{ Terms } & \multirow[t]{2}{*}{ Ref. } \\
\hline & & Obs. & Calc. & & & & \\
\hline 0.0549 & & 4.788 & 4.7878 & $0-20,886,130$ & $1 \mathrm{~s}^{2} 2 \mathrm{~s}-1 \mathrm{~s}\left({ }^{1} \mathrm{~S}\right) 2 \mathrm{~s} 4 \mathrm{p}\left({ }^{3} \mathrm{P}\right)$ & ${ }^{2} S_{1 / 2}-{ }^{2} P_{3 / 2}$ & {$[11]$} \\
\hline 0.0275 & & 4.788 & 4.7879 & $0-20,886,620$ & $1 \mathrm{~s}^{2} 2 \mathrm{~s}-1 \mathrm{~s}(\mathrm{~S}) 2 \mathrm{~s} 4 \mathrm{p}\left({ }^{3} \mathrm{P}\right)$ & $1 / 2$ & [11] \\
\hline 0.0071 & & 4.807 & 4.8033 & $21,027,320-208,204$ & $1 \mathrm{~s}(\mathrm{~S}) 2 \mathrm{p} 4 \mathrm{p}\left({ }^{1} \mathrm{P}\right)-1 \mathrm{~s}^{2} 2 \mathrm{p}$ & ${ }^{2} \mathrm{P}_{1 / 2}-$ & [11] \\
\hline 0.0247 & & 4.807 & 4.8059 & $21,027,320-219,430$ & $1 s^{1}(S) 2 p 4 p\left({ }^{1} P\right)-1 s^{2} 2 p$ & ${ }^{2} \mathrm{P}_{1 / 2}-$ & [11] \\
\hline 0.0064 & & 4.807 & 4.8053 & $21,018,640-208,204$ & $1 \mathrm{~s}\left({ }^{1} \mathrm{~S}\right) 2 \mathrm{p} 4 \mathrm{p}\left(^{3} \mathrm{P}\right)-1 \mathrm{~s}^{2} 2 \mathrm{p}$ & $\left({ }^{1} \mathrm{P}\right)^{2} \mathrm{D}_{3 / 2}$ & [11] \\
\hline 0.0209 & & 4.807 & 4.8077 & $21,019,240-219,430$ & $1 s(S) 2 p 4 p\left(^{3} P\right)-1 s^{2} 2 p$ & $\left({ }^{1} \mathrm{P}\right)^{2} \mathrm{D}_{5 / 2}$ & [11] \\
\hline 0.1369 & & 5.0126 & 5.0126 & $0-19,949,895$ & $1 s^{2}\left({ }^{1} S\right) 2 s-1 s\left({ }^{1} S\right) 2 s 3 p\left({ }^{3} P\right)$ & ${ }^{2} \mathrm{~S}_{1 / 2}-$ & [11] \\
\hline 0.0685 & & 5.0126 & 5.0123 & $0-19,951,015$ & $1 \mathrm{~s}^{2}\left({ }^{1} \mathrm{~S}\right) 2 \mathrm{~s}-1 \mathrm{~s}\left({ }^{1} \mathrm{~S}\right) 2 \mathrm{~s} 3 \mathrm{p}\left({ }^{3} \mathrm{P}\right)$ & ${ }^{2} \mathrm{~S}_{1 / 2}-{ }^{2} \mathrm{P}_{1 / 2}$ & [11] \\
\hline 0.0006 & & 5.0199 & 5.0196 & $0-19,921,845$ & $1 s^{2} 2 s-1 s\left({ }^{1} S\right) 2 s 3 p\left({ }^{1} P\right)$ & $\left({ }^{3} \mathrm{P}\right)^{2} \mathrm{~S}_{1 / 2}$ & [11] \\
\hline 0.0001 & & 5.0199 & 5.0199 & $0-19,920,720$ & $1 \mathrm{~s}^{2} 2 \mathrm{~s}-1 \mathrm{~s}\left({ }^{1} \mathrm{~S}\right) 2 \mathrm{~s} 3 \mathrm{p}\left({ }^{1} \mathrm{P}\right)$ & $\left({ }^{3} \mathrm{P}\right)^{2} \mathrm{~S}_{1 / 2}-{ }^{4} \mathrm{P}_{1 / 2}$ & [11] \\
\hline 0.0191 & & 5.0395 & 5.0422 & $20,040,625-208,204$ & $1 s\left({ }^{1} S\right) 2 p 3 p\left({ }^{1} P\right)-1 s^{2} 2 p$ & $\left({ }^{1} \mathrm{P}\right)^{2} \mathrm{~S}_{1 / 2}-{ }^{2} \mathrm{P}_{1 / 2}$ & [11] \\
\hline 0.0396 & & 5.0395 & 5.0451 & $20,040,625-219,430$ & $1 s\left({ }^{1} S\right) 2 p 3 p\left(\left(^{1} P\right)-1 s^{2} 2 p\right.$ & $\left({ }^{1} \mathrm{P}\right)^{2} \mathrm{~S}_{1 / 2}-{ }^{2} \mathrm{P}_{3 / 2}$ & [11] \\
\hline 0.1704 & & 5.0395 & 5.0479 & $20,029,775-219,430$ & $1 s\left({ }^{1} S\right) 2 p 3 p\left({ }^{1} P\right)-1 s^{2} 2 p$ & $\left({ }^{1} \mathrm{P}\right)^{2} \mathrm{D}_{5 / 2}-{ }^{2} \mathrm{P}_{3 / 2}$ & {$[11][19]$} \\
\hline 0.0585 & & 5.7836 & 5.7836 & $17,498,570-208,204$ & $1 s 2 p^{2}-1 s^{2} 2 p$ & $\mathrm{~S}_{1 / 2}-$ & {$[11]$} \\
\hline 0.1803 & & 5.7874 & 5.7873 & $17,498,570-219,430$ & $1 s 2 p^{2}-1 s^{2} 2 p$ & ${ }^{2} \mathrm{~S}_{1 / 2}-$ & {$[11]$} \\
\hline 0.0702 & & 5.7923 & 5.7923 & $0-17,264,350$ & $1 \mathrm{~s}^{2} 2 \mathrm{~s}-1 \mathrm{~s}\left({ }^{1} \mathrm{~S}\right) 2 \mathrm{~s} 2 \mathrm{p}\left({ }^{1} \mathrm{P}\right)$ & ${ }^{2} \mathrm{~S}_{1 / 2}-$ & [11] \\
\hline 0.0688 & & 5.7933 & 5.7934 & $0-17,261,115$ & $1 s^{2} 2 s-1 s\left({ }^{1} S\right) 2 s 2 p\left({ }^{1} P\right)$ & ${ }^{2} \mathrm{~S}_{1 / 2}-{ }^{2} \mathrm{P}_{1 / 2}$ & [11] \\
\hline 0.9818 & & 5.8169 & 5.8167 & $0-17,191,815$ & $1 s^{2} 2 s-1 s\left({ }^{1} S\right) 2 s 2 p\left({ }^{3} P\right)$ & ${ }^{2} \mathrm{~S}_{1 / 2}-$ & [11] \\
\hline 0.4574 & & 5.8169 & 5.8184 & $0-17,186,895$ & $1 \mathrm{~s}^{2} 2 \mathrm{~s}-1 \mathrm{~s}\left({ }^{1} \mathrm{~S}\right) 2 \mathrm{~s} 2 \mathrm{p}\left({ }^{3} \mathrm{P}\right)$ & $\mathrm{S}_{1 / 2}$ & [11] \\
\hline 0.0019 & & 5.0395 & 5.0422 & $20,040,625-208,204$ & $1 s\left({ }^{1} S\right) 2 p 3 p\left({ }^{1} P\right)-1 s^{2} 2 p$ & $\left({ }^{1} \mathrm{P}\right)^{2} \mathrm{~S}_{1 / 2}-{ }^{2} \mathrm{P}_{1 / 2}$ & [11] \\
\hline 0.5428 & & 5.8230 & 5.8240 & $17,378,605-208,204$ & $1 s 2 p^{2}-1 s^{2} 2 p$ & ${ }^{2} \mathrm{P}_{1 / 2}-{ }^{2} \mathrm{P}_{1 / 2}$ & [11] \\
\hline 1.3817 & & 5.8230 & 5.8245 & $17,388,180-219,430$ & $1 s 2 p^{2}-1 s^{2} 2 p$ & ${ }^{2} \mathrm{P}_{3 / 2}-{ }^{2} \mathrm{P}_{3 / 2}$ & {$[11]$} \\
\hline 0.0000 & & 5.8316 & ${ }^{*} 5.8321$ & $37,400,400-20,886,620$ & $1 s^{2} 4 s-1 s 2 s 4 p$ & ${ }^{2} \mathrm{~S}_{1 / 2}-{ }^{2} \mathrm{P}_{1 / 2}$ & {$[11]$} \\
\hline 0.0000 & & 5.8316 & ${ }^{*} 5.8322$ & $37,400,400-20,886,130$ & $1 s^{2} 4 s-1 s 2 s 4 p$ & ${ }^{2} \mathrm{~S}_{1 / 2}-{ }^{2} \mathrm{P}_{3 / 2}$ & [11] \\
\hline 0.0000 & & 5.8316 & ${ }^{*} 5.8322$ & $20,920,465-37,742,400$ & $1 \mathrm{~s} 2 \mathrm{p} 4 \mathrm{p}-1 \mathrm{~s}^{2} 4 \mathrm{f}$ & ${ }^{4} \mathrm{D}_{5 / 2}-{ }^{2} \mathrm{~F}_{5 / 2}$ & [11] \\
\hline 0.0000 & & 5.8316 & ${ }^{*} 5.8322$ & $20,920,465-37,743,800$ & $1 \mathrm{~s} 2 \mathrm{p} 4 \mathrm{p}-1 \mathrm{~s}^{2} 4 \mathrm{f}$ & ${ }^{4} \mathrm{D}_{5 / 2}-{ }^{2} \mathrm{~F}_{7 / 2}$ & [11] \\
\hline 0.5274 & & 5.8316 & 5.8324 & $17,353,875-208,204$ & $1 s 2 p^{2}-1 s^{2} 2 p$ & ${ }^{2} \mathrm{D}_{3 / 2}-$ & [11] \\
\hline 0.0109 & & 5.8361 & 5.8361 & $17,353,875-219,430$ & $1 s 2 p^{2}-1 s^{2} 2 p$ & ${ }^{2} \mathrm{D}_{3 / 2}-{ }^{2} \mathrm{P}_{3 / 2}$ & {$[11]$} \\
\hline 0.7635 & & 5.8365 & 5.8358 & $17,355,065-219,430$ & $1 s 2 p^{2}-1 s^{2} 2 p$ & ${ }^{2} \mathrm{D}_{5 / 2}-{ }^{2} \mathrm{P}_{3 / 2}$ & [11] \\
\hline 0.0000 & & 5.8365 & *5.8366 & $2,788,650-19,921,845$ & $1 \mathrm{~s} 3 \mathrm{~s}-1 \mathrm{~s} 2 \mathrm{~s} 3 \mathrm{p}$ & ${ }^{2} S_{1 / 2}-{ }^{4} P_{1 / 2}$ & [11] \\
\hline 0.0000 & & 5.8365 & ${ }^{*} 5.8370$ & $2,788,650-19,920,720$ & $1 s 3 s-1 s 2 s 3 p$ & ${ }^{2} \mathrm{~S}_{1 / 2}-{ }^{4} \mathrm{P}_{3 / 2}$ & [11] \\
\hline 0.0000 & & 5.869 & 5.8675 & $17,251,290-208,204$ & $1 s 2 p^{2}-1 s^{2} 2 p$ & ${ }^{4} \mathrm{P}_{3 / 2}-{ }^{2} \mathrm{P}_{1 / 2}$ & [11] \\
\hline 0.0005 & & 5.869 & 5.8689 & $17,246,905-208,204$ & $1 s 2 p^{2}-1 s^{2} 2 p$ & ${ }^{4} \mathrm{P}_{1 / 2}-{ }^{2} \mathrm{P}_{1 / 2}$ & [11] \\
\hline 0.0028 & & 5.869 & 5.8692 & $17,257,990-219,430$ & $1 s 2 p^{2}-1 s^{2} 2 p$ & ${ }^{4} \mathrm{P}_{5 / 2}-{ }^{2} \mathrm{P}_{3 / 2}$ & {$[11]$} \\
\hline 0.0010 & & 5.869 & 5.8713 & $17,251,290-219,430$ & $1 s 2 p^{2}-1 s^{2} 2 p$ & ${ }^{4} P_{3 / 2}-{ }^{2} P_{3 / 2}$ & [11] \\
\hline 0.0000 & & 5.869 & 5.8729 & $17,246,905-219,430$ & $1 s 2 p^{2}-1 s^{2} 2 p$ & ${ }^{4} P_{1 / 2}-{ }^{2} P_{3 / 2}$ & [11] \\
\hline 0.0002 & & 5.869 & ${ }^{*} 5.8726$ & $0-17,028,110$ & $1 \mathrm{~s} 2 \mathrm{~s}-1 \mathrm{~s} 2 \mathrm{~s} 2 \mathrm{p}$ & ${ }^{2} \mathrm{~S}_{1 / 2}-{ }^{4} \mathrm{P}_{3 / 2}$ & [11] \\
\hline
\end{tabular}




\begin{tabular}{|c|c|c|c|c|c|c|c|}
\hline \multicolumn{8}{|c|}{ Continued } \\
\hline 0.0009 & & 5.869 & ${ }^{*} 5.8740$ & $0-17,024,200$ & $1 s 2 s-1 s 2 s 2 p$ & ${ }^{2} \mathrm{~S}_{1 / 2}-{ }^{4} \mathrm{P}_{1 / 2}$ & {$[11]$} \\
\hline 0.0284 & 1 & $22.64 / .678$ & 22.6545 & $4,633,565-219,430$ & $1 \mathrm{~s}^{2} 8 \mathrm{~d}-1 \mathrm{~s}^{2} 2 \mathrm{p}$ & ${ }^{2} \mathrm{D}_{5 / 2}-{ }^{2} \mathrm{P}_{3 / 2}$ & {$[4][5]$} \\
\hline 0.0032 & 1 & $22.64 / .678^{*}$ & 22.6549 & $4,633,486-219,430$ & $1 \mathrm{~s}^{2} 8 \mathrm{~d}-1 \mathrm{~s}^{2} 2 \mathrm{p}$ & ${ }^{2} \mathrm{D}_{3 / 2}-{ }^{2} \mathrm{P}_{3 / 2}$ & {$[4][5]$} \\
\hline 0.0452 & 1 & $23.08 / .075$ & 23.0752 & $4,553,085-219,430$ & $1 \mathrm{~s}^{2} 7 \mathrm{~d}-1 \mathrm{~s}^{2} 2 \mathrm{p}$ & ${ }^{2} \mathrm{D}_{5 / 2}$ & [4] [5] \\
\hline 0.0050 & 1 & $23.08 / .075$ & ${ }^{*} 23.0764$ & $4,552,860-219,430$ & $1 \mathrm{~s}^{2} 7 \mathrm{~d}-1 \mathrm{~s}^{2} 2 \mathrm{p}$ & ${ }^{2} \mathrm{D}_{3 / 2}-{ }^{2} \mathrm{P}_{3 / 2}$ & {$[4][5]$} \\
\hline 0.0440 & 50 & 23.75 & 23.7465 & $4,419,350-208,204$ & $1 s^{2} 6 d-1 s^{2} 2 p$ & ${ }^{2} \mathrm{D}_{3 / 2}-$ & {$[5]$} \\
\hline $\begin{array}{c}0.0790 \\
50\end{array}$ & 50 & 23.810 & 23.8100 & $4,419,350-219,430$ & $1 s^{2} 6 d-1 s^{2} 2 p$ & ${ }^{2} \mathrm{D}_{5 / 2}$ & {$[5]$} \\
\hline $\begin{array}{c}0.0088 \\
50\end{array}$ & 50 & 23.810 & 23.8100 & $4,419,350-219,430$ & $1 \mathrm{~s}^{2} 6 \mathrm{~d}-1 \mathrm{~s}^{2} 2 \mathrm{p}$ & ${ }^{2} \mathrm{D}_{3 / 2}$ & {$[5]$} \\
\hline 0.0899 & 3 & 25.103 & 25.0998 & $4,192,300-208,204$ & $1 \mathrm{~s}^{2} 5 \mathrm{~d}-1 \mathrm{~s}^{2} 2 \mathrm{p}$ & ${ }^{2} \mathrm{D}_{3 / 2}$ & {$[5]$} \\
\hline 0.1613 & 4 & 25.169 & 25.1707 & $4,192,300-219,430$ & $1 \mathrm{~s}^{2} 5 \mathrm{~d}-1 \mathrm{~s}^{2} 2 \mathrm{p}$ & ${ }^{2} \mathrm{D}_{5 / 2}$ & {$[5]$} \\
\hline 0.0179 & 4 & 25.169 & 25.1707 & $4,1923,00-219,430$ & $1 \mathrm{~s}^{2} 5 \mathrm{~d}-1 \mathrm{~s}^{2} 2 \mathrm{p}$ & ${ }^{2} \mathrm{D}_{3 / 2}-{ }^{2} \mathrm{P}_{3 / 2}$ & [5] \\
\hline 0.0033 & 4 & 25.169 & *25.1693 & $4,181,300-208,204$ & $1 \mathrm{~s}^{2} 5 \mathrm{~s}-1 \mathrm{~s}^{2} 2 \mathrm{p}$ & ${ }^{2} \mathrm{~S}_{1 / 2}-{ }^{2} \mathrm{P}_{1 / 2}$ & {$[5]$} \\
\hline 0.1219 & 5 & 26.608 & 26.6087 & $0-3,758,169$ & $1 s^{2} 2 s-1 s^{2} 4 p$ & ${ }^{2} \mathrm{~S}_{1 / 2}-{ }^{2} \mathrm{P}_{3 / 2}$ & {$[5]$} \\
\hline 0.0609 & 5 & 26.608 & 26.6091 & $0-3,758,112$ & $1 s^{2} 2 s-1 s^{2} 4 p$ & ${ }^{2} \mathrm{~S}_{1 / 2}-$ & [5] \\
\hline 0.2435 & 6 & 28.044 & 28.0482 & $3,773,500-208,204$ & $1 s^{2} 4 d-1 s^{2} 2 p$ & ${ }^{2} \mathrm{D}_{3 / 2}$ & {$[5]$} \\
\hline 0.4369 & 6 & 28.128 & 28.1328 & $3,774,000-219,430$ & $1 \mathrm{~s}^{2} 4 \mathrm{~d}-1 \mathrm{~s}^{2} 2 \mathrm{p}$ & ${ }^{2} \mathrm{D}_{5 / 2}$ & [5] \\
\hline 0.0485 & 6 & 28.128 & ${ }^{*} 28.1368$ & $3,773,500-219,430$ & $1 s^{2} 4 d-1 s^{2} 2 p$ & ${ }^{2} \mathrm{D}_{3 / 2}-{ }^{2} \mathrm{P}_{3 / 2}$ & {$[16][17]$} \\
\hline 0.0085 & 2 & 28.337 & ${ }^{*} 28.3139$ & $3,740,040-208,204$ & $1 s^{2} 4 s-1 s^{2} 2 p$ & ${ }^{2} \mathrm{~S}_{1 / 2}$ & {$[5]$} \\
\hline 0.0169 & 2 & 28.337 & ${ }^{*} 28.4042$ & $3,740,040-219,430$ & $1 \mathrm{~s}^{2} 4 \mathrm{~s}-1 \mathrm{~s}^{2} 2 \mathrm{p}$ & ${ }^{2} \mathrm{~S}_{1 / 2}-$ & [5] \\
\hline 0.4817 & 5 & 35.095 & 35.0950 & $0-2,849,410$ & $1 s^{2} 2 s-1 s^{2} 3 p$ & ${ }^{2} \mathrm{~S}_{1 / 2}-{ }^{2} \mathrm{P}_{3 / 2}$ & [5] \\
\hline 0.2406 & 7 & 35.136 & 35.1361 & $0-2,846,080$ & $1 s^{2} 2 s-1 s^{2} 3 p$ & ${ }^{2} \mathrm{~S}_{1 / 2}-$ & {$[5]$} \\
\hline 1.3341 & 7 & 37.561 & 37.5610 & $2,870,540-208,204$ & $1 \mathrm{~s}^{2} 3 \mathrm{~d}-1 \mathrm{~s}^{2} 2 \mathrm{p}$ & ${ }^{2} \mathrm{D}_{3 / 2}-{ }^{2} \mathrm{P}_{1 / 2}$ & {$[5]$} \\
\hline 2.3922 & 8 & 37.706 & 37.7060 & $2,871,530-219,430$ & $1 s^{2} 3 d-1 s^{2} 2 p$ & ${ }^{2} \mathrm{D}_{5 / 2}-{ }^{2} \mathrm{P}_{3 / 2}$ & {$[5]$} \\
\hline 0.2657 & 0 & 37.723 & 37.7201 & $2,870,540-219,430$ & $1 \mathrm{~s}^{2} 3 \mathrm{~d}-1 \mathrm{~s}^{2} 2 \mathrm{p}$ & ${ }^{2} \mathrm{D}_{3 / 2}-$ & {$[5]$} \\
\hline 0.0397 & 3 & 38.754 & 38.7530 & $2,788,650-208,204$ & $1 \mathrm{~s}^{2} 3 \mathrm{~s}-1 \mathrm{~s}^{2} 2 \mathrm{p}$ & ${ }^{2} \mathrm{~S} 1_{/ 2}-{ }^{2} \mathrm{P}_{1 / 2}$ & {$[5]$} \\
\hline 0.0791 & 3 & 38.921 & 38.9223 & $2,788,650-219,430$ & $1 s^{2} 3 s-1 s^{2} 2 p$ & ${ }^{2} \mathrm{~S}_{1 / 2}-{ }^{2} \mathrm{P}_{3 / 2}$ & {$[5]$} \\
\hline 0.2693 & 1 & 74.357 & 74.2820 & $4,192,300-2,846,080$ & $1 \mathrm{~s}^{2} 5 \mathrm{~d}-1 \mathrm{~s}^{2} 3 \mathrm{p}$ & ${ }^{2} \mathrm{D}_{3 / 2}-{ }^{2} \mathrm{P}_{1 / 2}$ & {$[5]$} \\
\hline 0.0537 & 2 & 74.563 & ${ }^{*} 74.4662$ & $4,192,300-2,849,410$ & $1 \mathrm{~s}^{2} 5 \mathrm{~d}-1 \mathrm{~s}^{2} 3 \mathrm{p}$ & ${ }^{2} \mathrm{D}_{3 / 2}-{ }^{2} \mathrm{P}_{3 / 2}$ & {$[5]$} \\
\hline 0.4836 & 2 & 74.563 & 74.4662 & $4,192,300-2,849,410$ & $1 \mathrm{~s}^{2} 5 \mathrm{~d}-1 \mathrm{~s}^{2} 3 \mathrm{p}$ & ${ }^{2} \mathrm{D}_{5 / 2}-{ }^{2} 3 / 2$ & {$[5]$} \\
\hline 0.1331 & 10 & $455.67 / .73$ & 455.7256 & $0-219,430$ & $1 \mathrm{~s}^{2} 2 \mathrm{~s}-1 \mathrm{~s}^{2} 2 \mathrm{p}$ & ${ }^{2} \mathrm{~S} 1_{/ 2}-{ }^{2} \mathrm{P} 5_{/ 2}$ & {$[6]-[8]$} \\
\hline 0.6310 & - & $480.42 / .298$ & 480.2973 & $0-208,204$ & $1 \mathrm{~s}^{2} 2 \mathrm{~s}-1 \mathrm{~s}^{2} 2 \mathrm{p}$ & ${ }^{2} \mathrm{~S}_{1 / 2}-{ }^{2} \mathrm{P}_{1 / 2}$ & {$[6]-[8]$} \\
\hline 0.2259 & - & 1645.8 & 1645.8343 & $2,788,650-2,849,410$ & $1 s^{2} 3 s-1 s^{2} 3 p$ & ${ }^{2} \mathrm{~S}_{1 / 2}-{ }^{2} \mathrm{P}_{3 / 2}$ & {$[15][16]$} \\
\hline 0.1068 & - & 1741.3 & 1741.2672 & $2,788,650-2,846,080$ & $1 \mathrm{~s}^{2} 3 \mathrm{~s}-1 \mathrm{~s}^{2} 3 \mathrm{p}$ & ${ }^{2} \mathrm{~S}_{1 / 2}-{ }^{2} \mathrm{P}_{1 / 2}$ & {$[15][16]$} \\
\hline 0.3068 & - & 4087. & 4079.096 & $3,740,040-3,764,550$ & $1 s^{2} 4 s-1 s^{2} 4 p$ & ${ }^{2} \mathrm{~S}_{1 / 2}-{ }^{2} \mathrm{P}_{3 / 2}$ & {$[15][16]$} \\
\hline 0.0969 & - & 4520. & 4519.470 & $2,871,530-2,849,410$ & $1 \mathrm{~s}^{2} 3 \mathrm{~d}-1 \mathrm{~s}^{2} 3 \mathrm{p}$ & ${ }^{2} \mathrm{D}_{5 / 2}-{ }^{2} \mathrm{P}_{3 / 2}$ & {$[15][16]$} \\
\hline 0.0103 & - & 4731. & 4731.222 & $2,870,540-2,849,410$ & $1 \mathrm{~s}^{2} 3 \mathrm{~d}-1 \mathrm{~s}^{2} 3 \mathrm{p}$ & ${ }^{2} \mathrm{D}_{3 / 2}-{ }^{2} \mathrm{P}_{3 / 2}$ & {$[15][16]$} \\
\hline
\end{tabular}


Table 2. (a) Even configurations. (b) Odd configurations.

(a)

\begin{tabular}{|c|c|c|c|c|}
\hline Lifetimes (s) & Energy levels $\left(\mathrm{cm}^{-1}\right)$ & Configurations & Terms & Percentage Composition \\
\hline & 0 & $1 \mathrm{~s}^{2} 2 \mathrm{~s}$ & ${ }^{2} \mathrm{~S}_{1 / 2}$ & $100 \%$ \\
\hline $3.812 \times 10^{-12}$ & $2,788,650$ & $1 \mathrm{~s}^{2} 3 \mathrm{~s}$ & ${ }^{2} \mathrm{~S} 1 / 2$ & $100 \%$ \\
\hline $5.296 \times 10^{-13}$ & $2,870,540$ & $1 \mathrm{~s}^{2} 3 \mathrm{~d}$ & ${ }^{2} \mathrm{D} 3 / 2$ & $100 \%$ \\
\hline $5.346 \times 10^{-13}$ & $2,871,530$ & $1 \mathrm{~s}^{2} 3 \mathrm{~d}$ & ${ }^{2}$ D5/2 & $100 \%$ \\
\hline $5.693 \times 10^{-12}$ & $3,740,040$ & $1 \mathrm{~s}^{2} 4 \mathrm{~s}$ & ${ }^{2} \mathrm{~S} 1 / 2$ & $100 \%$ \\
\hline $1.229 \times 10^{-12}$ & $3,773,500$ & $1 \mathrm{~s}^{2} 4 \mathrm{~d}$ & ${ }^{2} \mathrm{D} 3 / 2$ & $100 \%$ \\
\hline $1.239 \times 10^{-12}$ & $3,774,000$ & $1 \mathrm{~s}^{2} 4 \mathrm{~d}$ & ${ }^{2} \mathrm{D} 5 / 2$ & $100 \%$ \\
\hline $8.880 \times 10^{-12}$ & $4,181,300$ & $1 \mathrm{~s}^{2} 5 \mathrm{~s}$ & ${ }^{2} \mathrm{~S} 1 / 2$ & $100 \%$ \\
\hline $3.508 \times 10^{-12}$ & $4,192,300$ & $1 \mathrm{~s}^{2} 5 \mathrm{~d}$ & ${ }^{2} \mathrm{D} 3 / 2$ & $100 \%$ \\
\hline $3.533 \times 10^{-12}$ & $4,192,300$ & $1 \mathrm{~s}^{2} 5 \mathrm{~d}$ & ${ }^{2} \mathrm{D} 5 / 2$ & $100 \%$ \\
\hline $7.800 \times 10^{-12}$ & ${ }^{*} 4,199,270$ & $1 \mathrm{~s}^{2} 5 \mathrm{~g}$ & ${ }^{2} \mathrm{G} 5 / 2$ & $100 \%$ \\
\hline $7.804 \times 10^{-12}$ & ${ }^{*} 4,199,330$ & $1 \mathrm{~s}^{2} 5 \mathrm{~g}$ & ${ }^{2} \mathrm{G} 7 / 2$ & $100 \%$ \\
\hline $1.379 \times 10^{-11}$ & ${ }^{*} 4,415,640$ & $1 \mathrm{~s}^{2} 6 \mathrm{~s}$ & ${ }^{2} \mathrm{~S} 1 / 2$ & $100 \%$ \\
\hline $6.412 \times 10^{-12}$ & $4,419,350$ & $1 \mathrm{~s}^{2} 6 \mathrm{~d}$ & ${ }^{2} \mathrm{D} 3 / 2$ & $100 \%$ \\
\hline $6.455 \times 10^{-12}$ & $4,419,350$ & $1 \mathrm{~s}^{2} 6 \mathrm{~d}$ & ${ }^{2} \mathrm{D} 5 / 2$ & $100 \%$ \\
\hline $1.384 \times 10^{-11}$ & $* 4,425,975$ & $1 \mathrm{~s}^{2} 6 \mathrm{~g}$ & ${ }^{2} \mathrm{G} 5 / 2$ & $100 \%$ \\
\hline $1.384 \times 10^{-11}$ & ${ }^{*} 4,426,010$ & $1 \mathrm{~s}^{2} 6 \mathrm{~g}$ & ${ }^{2} \mathrm{G} 7 / 2$ & $100 \%$ \\
\hline $2.046 \times 10^{-11}$ & ${ }^{*} 4,556,200$ & $1 \mathrm{~s}^{2} 7 \mathrm{~s}$ & ${ }^{2} \mathrm{~S} 1 / 2$ & $100 \%$ \\
\hline $6.284 \times 10^{-12}$ & $* 4,552,860$ & $1 \mathrm{~s}^{2} 7 \mathrm{~d}$ & ${ }^{2} \mathrm{D} 3 / 2$ & $100 \%$ \\
\hline $6.319 \times 10^{-12}$ & $4,553,085$ & $1 \mathrm{~s}^{2} 7 \mathrm{~d}$ & ${ }^{2} \mathrm{D} 5 / 2$ & $100 \%$ \\
\hline $2.916 \times 10^{-11}$ & ${ }^{*} 4,647,077$ & $1 \mathrm{~s}^{2} 8 \mathrm{~s}$ & ${ }^{2} \mathrm{~S} 1 / 2$ & $100 \%$ \\
\hline $9.419 \times 10^{-12}$ & ${ }^{*} 4,633,445$ & $1 \mathrm{~s}^{2} 8 \mathrm{~d}$ & ${ }^{2} \mathrm{D} 5 / 2$ & $100 \%$ \\
\hline $9.369 \times 10^{-12}$ & $4,633,565$ & $1 \mathrm{~s}^{2} 8 \mathrm{~d}$ & ${ }^{2} \mathrm{D} 5 / 2$ & $100 \%$ \\
\hline $4.314 \times 10^{-13}$ & *16,971,300 & $1 s s^{2}$ & $\left({ }^{2} \mathrm{~S}\right)^{2} \mathrm{~S} 1 / 2$ & $92 \%+8 \% 1 \mathrm{~s} 2 \mathrm{p}^{2}(\mathrm{~S})^{1} \mathrm{~S}$ \\
\hline $2.060 \times 10^{-11}$ & $17,246,905$ & $1 \mathrm{~s} 2 \mathrm{p}^{2}$ & $\left({ }^{3} \mathrm{P}\right)^{4} \mathrm{P} 1 / 2$ & $100 \%$ \\
\hline $1.987 \times 10^{-11}$ & $17,251,290$ & $1 \mathrm{~s} 2 \mathrm{p}^{2}$ & $\left({ }^{3} \mathrm{P}\right)^{4} \mathrm{P} 3 / 2$ & $100 \%$ \\
\hline $1.075 \times 10^{-11}$ & $17,257,990$ & $1 \mathrm{~s} 2 \mathrm{p}^{2}$ & $\left({ }^{3} \mathrm{P}\right)^{4} \mathrm{P} 5 / 2$ & $100 \%$ \\
\hline $3.789 \times 10^{-14}$ & $17,353,875$ & $1 \mathrm{~s} 2 \mathrm{p}^{2}$ & $\left({ }^{1} \mathrm{D}\right)^{2} \mathrm{D} 3 / 2$ & $97 \%$ \\
\hline $4.011 \times 10^{-14}$ & $17,355,065$ & $1 \mathrm{~s} 2 \mathrm{p}^{2}$ & $(\mathrm{l})^{2} \mathrm{D} 5 / 2$ & $100 \%$ \\
\hline $1.299 \times 10^{-14}$ & $17,378,605$ & $1 \mathrm{~s} 2 \mathrm{p}^{2}$ & $\left({ }^{3} \mathrm{P}\right)^{2} \mathrm{P} 1 / 2$ & $100 \%$ \\
\hline $1.319 \times 10^{-14}$ & $17,388,180$ & $1 \mathrm{~s} 2 \mathrm{p}^{2}$ & $\left({ }^{3} \mathrm{P}\right)^{2} \mathrm{P} 3 / 2$ & $97 \%$ \\
\hline $4.198 \times 10^{-14}$ & $17,498,570$ & $1 \mathrm{~s} 2 \mathrm{p}^{2}$ & $\left({ }^{1} \mathrm{~S}\right)^{2} \mathrm{~S} 1 / 2$ & $91 \%+8 \% 1 \mathrm{~s}^{2} \mathrm{~s}^{2}(\mathrm{~S})$ \\
\hline
\end{tabular}




\section{Continued}

\begin{tabular}{|c|c|c|c|c|}
\hline $1.422 \times 10^{-12}$ & ${ }^{*} 19,974,700$ & $1 \mathrm{~s} 2 \mathrm{p} 3 \mathrm{p}$ & $\left({ }^{3} \mathrm{P}\right)^{4} \mathrm{D} 1 / 2$ & $95 \%$ \\
\hline $1.484 \times 10^{-12}$ & $* 19,797,615$ & $1 \mathrm{~s} 2 \mathrm{p} 3 \mathrm{p}$ & $\left({ }^{3} \mathrm{P}\right)^{4} \mathrm{D} 3 / 2$ & $96 \%$ \\
\hline $1.612 \times 10^{-12}$ & $* 19,802,455$ & $1 \mathrm{~s} 2 \mathrm{p} 3 \mathrm{p}$ & $\left({ }^{3} \mathrm{P}\right)^{4} \mathrm{D} 5 / 2$ & $99 \%$ \\
\hline $1.656 \times 10^{-12}$ & $* 10,809,080$ & $1 \mathrm{~s} 2 \mathrm{p} 3 \mathrm{p}$ & $\left({ }^{3} \mathrm{P}\right)^{4} \mathrm{D} 7 / 2$ & $100 \%$ \\
\hline $1.289 \times 10^{-12}$ & ${ }^{*} 19,816,705$ & $1 \mathrm{~s} 2 \mathrm{p} 3 \mathrm{p}$ & $\left({ }^{3} \mathrm{P}\right)^{4} \mathrm{~S} 3 / 2$ & $56 \%+39 \% 1 \mathrm{~s} 2 \mathrm{p} 3 \mathrm{p}\left({ }^{3} \mathrm{P}\right)^{2} \mathrm{P}$ \\
\hline $5.117 \times 10^{-14}$ & $* 19,867,315$ & $1 \mathrm{~s} 2 \mathrm{p} 3 \mathrm{p}$ & $\left({ }^{3} \mathrm{P}\right)^{2} \mathrm{P} 3 / 2$ & $50 \%+41 \% 1 \mathrm{~s} 2 \mathrm{p} 3 \mathrm{p}\left({ }^{3} \mathrm{P}\right)^{4} \mathrm{~S}$ \\
\hline $5.114 \times 10^{-14}$ & $* 19,867,440$ & $1 \mathrm{~s} 2 \mathrm{p} 3 \mathrm{p}$ & $\left({ }^{3} \mathrm{P}\right)^{2} \mathrm{P} 1 / 2$ & $89 \%+5 \% 1 \mathrm{~s} 2 \mathrm{p} 3 \mathrm{p}\left({ }^{3} \mathrm{P}\right)^{4} \mathrm{D}+5 \% 1 \mathrm{~s} 2 \mathrm{p} 3 \mathrm{p}\left({ }^{1} \mathrm{P}\right)^{2} \mathrm{P}$ \\
\hline $1.850 \times 10^{-14}$ & $* 20,021,950$ & $1 \mathrm{~s} 2 \mathrm{p} 3 \mathrm{p}$ & $\left({ }^{1} \mathrm{P}\right)^{2} \mathrm{D} 3 / 2$ & $89 \%+9 \% 1 \mathrm{~s} 2 \mathrm{p} 3 \mathrm{p}\left({ }^{3} \mathrm{P}\right)^{2} \mathrm{D}$ \\
\hline $1.836 \times 10^{-14}$ & $20,029,775$ & $1 \mathrm{~s} 2 \mathrm{p} 3 \mathrm{p}$ & $\left({ }^{1} \mathrm{P}\right)^{2} \mathrm{D} 5 / 2$ & $94 \%+6 \% 1 \mathrm{~s} 2 \mathrm{p} 3 \mathrm{p}\left({ }^{3} \mathrm{P}\right)^{2} \mathrm{D}$ \\
\hline $1.818 \times 10^{-14}$ & $20,040,625$ & $1 \mathrm{~s} 2 \mathrm{p} 3 \mathrm{p}$ & $\left({ }^{1} \mathrm{P}\right)^{2} \mathrm{~S}_{1 / 2}$ & $79 \%+19 \% 1 \mathrm{~s} 2 \mathrm{p} 3 \mathrm{p}\left(^{3} \mathrm{P}\right)^{2} \mathrm{~S}$ \\
\hline $9.880 \times 10^{-12}$ & *20,322,915 & $1 \mathrm{~s} 2 \mathrm{p} 3 \mathrm{p}$ & $\left({ }^{3} \mathrm{P}\right){ }^{4} \mathrm{P} 1 / 2$ & $99 \%$ \\
\hline $1.002 \times 10^{-12}$ & ${ }^{*} 20,325,950$ & $1 \mathrm{~s} 2 \mathrm{p} 3 \mathrm{p}$ & $\left({ }^{3} \mathrm{P}\right){ }^{4} \mathrm{P} 3 / 2$ & $96 \%$ \\
\hline $9.924 \times 10^{-13}$ & $* 20,330,500$ & $1 \mathrm{~s} 2 \mathrm{p} 3 \mathrm{p}$ & $\left({ }^{3} \mathrm{P}\right)^{4} \mathrm{P} 5 / 2$ & $99 \%$ \\
\hline $7.210 \times 10^{-14}$ & *20,434,240 & $1 \mathrm{~s} 2 \mathrm{p} 3 \mathrm{p}$ & $\left({ }^{3} \mathrm{P}\right)^{2} \mathrm{D} 3 / 2$ & $89 \%+9 \% 1 \mathrm{~s} 2 \mathrm{p} 3 \mathrm{p}\left({ }^{1} \mathrm{P}\right)^{2} \mathrm{D}$ \\
\hline $7.442 \times 10^{-14}$ & *20,435,930 & $1 \mathrm{~s} 2 \mathrm{p} 3 \mathrm{p}$ & $\left({ }^{3} \mathrm{P}\right)^{2} \mathrm{D} 5 / 2$ & $93 \%+6 \% 1 \mathrm{~s} 2 \mathrm{p} 3 \mathrm{p}\left({ }^{1} \mathrm{P}\right)^{2} \mathrm{D}$ \\
\hline $1.818 \times 10^{-14}$ & ${ }^{*} 20,468,175$ & $1 \mathrm{~s} 2 \mathrm{p} 3 \mathrm{p}$ & $\left({ }^{3} \mathrm{P}\right)^{2} \mathrm{~S} 1 / 2$ & $80 \%+18 \% 1 \mathrm{~s} 2 \mathrm{p} 3 \mathrm{p}\left({ }^{1} \mathrm{P}\right)^{2} \mathrm{~S}$ \\
\hline $1.967 \times 10^{-14}$ & *20,559,010 & $1 \mathrm{~s} 2 \mathrm{p} 3 \mathrm{p}$ & $\left({ }^{1} \mathrm{P}\right)^{2} \mathrm{P} 1 / 2$ & $91 \%+6 \% 1 \mathrm{~s} 2 \mathrm{p} 3 \mathrm{p}\left({ }^{3} \mathrm{P}\right)^{2} \mathrm{P}$ \\
\hline $1.968 \times 10^{-14}$ & $* 20,564,875$ & $1 \mathrm{~s} 2 \mathrm{p} 3 \mathrm{p}$ & $\left({ }^{1} \mathrm{P}\right)^{2} \mathrm{P} 3 / 2$ & $91 \%+7 \% 1 \mathrm{~s} 2 \mathrm{p} 3 \mathrm{p}\left({ }^{3} \mathrm{P}\right)^{2} \mathrm{P}$ \\
\hline $1.479 \times 10^{-12}$ & ${ }^{*} 20,913,970$ & $1 \mathrm{~s} 2 \mathrm{p} 4 \mathrm{p}$ & $\left({ }^{3} \mathrm{P}\right)^{4} \mathrm{D} 1 / 2$ & $92 \%+7 \% 1 \mathrm{~s} 2 \mathrm{p} 4 \mathrm{p}\left({ }^{3} \mathrm{P}\right)^{2} \mathrm{P}$ \\
\hline $1.919 \times 10^{-12}$ & "20,916,430 & $1 \mathrm{~s} 2 \mathrm{p} 4 \mathrm{p}$ & $\left({ }^{3} \mathrm{P}\right)^{4} \mathrm{D} 3 / 2$ & $94 \%$ \\
\hline $2.164 \times 10^{-12}$ & $20,920,465$ & $1 \mathrm{~s} 2 \mathrm{p} 4 \mathrm{p}$ & $\left({ }^{3} \mathrm{P}\right)^{4} \mathrm{D} 5 / 2$ & $93 \%+6 \% 1 \mathrm{~s} 2 \mathrm{p} 4 \mathrm{p}\left({ }^{3} \mathrm{P}\right)^{4} \mathrm{P}$ \\
\hline $3.429 \times 10^{-12}$ & *20,927,445 & $1 \mathrm{~s} 2 \mathrm{p} 4 \mathrm{p}$ & $\left({ }^{3} \mathrm{P}\right)^{4} \mathrm{D} 7 / 2$ & $100 \%$ \\
\hline $5.873 \times 10^{-13}$ & "20,922,595 & $1 \mathrm{~s} 2 \mathrm{p} 4 \mathrm{p}$ & $\left({ }^{3} \mathrm{P}\right)^{4} \mathrm{~S} 3 / 2$ & $49 \%+25 \% 1 \mathrm{~s} 2 \mathrm{p} 4 \mathrm{p}\left({ }^{3} \mathrm{P}\right)^{2} \mathrm{P}+24 \% 1 \mathrm{~s} 2 \mathrm{p} 4 \mathrm{p}\left({ }^{3} \mathrm{P}\right)^{4} \mathrm{P}$ \\
\hline $2.679 \times 10^{-13}$ & *20,925,580 & $1 \mathrm{~s} 2 \mathrm{p} 4 \mathrm{p}$ & $\left({ }^{3} \mathrm{P}\right)^{2} \mathrm{P} 1 / 2$ & $74 \%+20 \% 1 \mathrm{~s} 2 \mathrm{p} 4 \mathrm{p}\left({ }^{3} \mathrm{P}\right){ }^{4} \mathrm{P}$ \\
\hline $7.846 \times 10^{-13}$ & *20,927,915 & $1 \mathrm{~s} 2 \mathrm{p} 4 \mathrm{p}$ & $\left({ }^{3} \mathrm{P}\right){ }^{4} \mathrm{P} 1 / 2$ & $77 \%+16 \% 1 \mathrm{~s} 2 \mathrm{p} 4 \mathrm{p}\left({ }^{3} \mathrm{P}\right)^{2} \mathrm{P}$ \\
\hline $2.711 \times 10^{-13}$ & *20,930,500 & $1 \mathrm{~s} 2 \mathrm{p} 4 \mathrm{p}$ & $\left({ }^{3} \mathrm{P}\right)^{2} \mathrm{P} 3 / 2$ & $60 \%+19 \% 1 \mathrm{~s} 2 \mathrm{p} 4 \mathrm{p}\left({ }^{3} \mathrm{P}\right)^{4} \mathrm{~S}+12 \% 1 \mathrm{~s} 2 \mathrm{p} 4 \mathrm{p}\left({ }^{3} \mathrm{P}\right)^{2} \mathrm{D}$ \\
\hline $2.952 \times 10^{-12}$ & *20,933,730 & $1 \mathrm{~s} 2 \mathrm{p} 4 \mathrm{p}$ & $(3 \mathrm{P})^{4} \mathrm{P} 3 / 2$ & $67 \%+31 \% 1 \mathrm{~s} 2 \mathrm{p} 4 \mathrm{p}\left({ }^{3} \mathrm{P}\right)^{4} \mathrm{~S}$ \\
\hline $2.444 \times 10^{-12}$ & *20,934,970 & $1 \mathrm{~s} 2 \mathrm{p} 4 \mathrm{p}$ & $\left({ }^{3} \mathrm{P}\right)^{4} \mathrm{P} 5 / 2$ & $92 \%+6 \% 1 \mathrm{~s} 2 \mathrm{p} 4 \mathrm{p}\left({ }^{3} \mathrm{P}\right){ }^{4} \mathrm{D}$ \\
\hline $1.880 \times 10^{-13}$ & *20,939,950 & $1 \mathrm{~s} 2 \mathrm{p} 4 \mathrm{p}$ & $\left({ }^{3} \mathrm{P}\right)^{2} \mathrm{D} 3 / 2$ & $85 \%+12 \% 1 \mathrm{~s} 2 \mathrm{p} 4 \mathrm{p}\left({ }^{3} \mathrm{P}\right)^{2} \mathrm{P}$ \\
\hline $2.190 \times 10^{-13}$ & *20,946,885 & $1 \mathrm{~s} 2 \mathrm{p} 4 \mathrm{p}$ & $\left({ }^{3} \mathrm{P}\right)^{2} \mathrm{D} 5 / 2$ & $97 \%$ \\
\hline $1.567 \times 10^{-13}$ & *20,956,590 & $1 \mathrm{~s} 2 \mathrm{p} 4 \mathrm{p}$ & $\left({ }^{3} \mathrm{P}\right)^{2} \mathrm{~S} 1 / 2$ & $94 \%$ \\
\hline $1.836 \times 10^{-14}$ & $21,018,640$ & $1 \mathrm{~s} 2 \mathrm{p} 4 \mathrm{p}$ & $\left({ }^{1} \mathrm{P}\right)^{2} \mathrm{D} 3 / 2$ & $97 \%$ \\
\hline $1.812 \times 10^{-14}$ & $21,019,240$ & $1 \mathrm{~s} 2 \mathrm{p} 4 \mathrm{p}$ & $\left({ }^{1} \mathrm{P}\right)^{2} \mathrm{D} 5 / 2$ & $100 \%$ \\
\hline $1.801 \times 10^{-14}$ & ${ }^{*} 21,020,865$ & $1 \mathrm{~s} 2 \mathrm{p} 4 \mathrm{p}$ & $(1 \mathrm{P})^{2} \mathrm{P} 1 / 2$ & $97 \%$ \\
\hline $1.810 \times 10^{-14}$ & *21,022,540 & $1 \mathrm{~s} 2 \mathrm{p} 4 \mathrm{p}$ & $\left({ }^{1} \mathrm{P}\right)^{2} \mathrm{P} 3 / 2$ & $97 \%$ \\
\hline $1.828 \times 10^{-14}$ & $21,027,320$ & $1 \mathrm{~s} 2 \mathrm{p} 4 \mathrm{p}$ & $\left({ }^{1} \mathrm{P}\right)^{2} \mathrm{~S} 1 / 2$ & $97 \%$ \\
\hline
\end{tabular}

( $^{*}$ Indicates an attempt to identify. 
(b)

\begin{tabular}{|c|c|c|c|c|}
\hline Lifetimes (s) & Energy levels $\left(\mathrm{cm}^{-1}\right)$ & Configurations & Terms & Leading Percentage \\
\hline $1.096 \times 10^{-9}$ & 208,204 & $1 \mathrm{~s}^{2} 2 \mathrm{p}$ & ${ }^{2} \mathrm{P} 1 / 2$ & $100 \%$ \\
\hline $9.360 \times 10^{-10}$ & 219,430 & $1 s^{2} 2 p$ & ${ }^{2} \mathrm{P} 3 / 2$ & $100 \%$ \\
\hline $1.539 \times 10^{-12}$ & $2,846,080$ & $1 \mathrm{~s}^{2} 3 \mathrm{p}$ & ${ }^{2} \mathrm{P} 1 / 2$ & $100 \%$ \\
\hline $1.533 \times 10^{-12}$ & $2,849,410$ & $1 s^{2} 3 p$ & ${ }^{2} \mathrm{P} 3 / 2$ & $100 \%$ \\
\hline $2.613 \times 10^{-12}$ & $3,756,701$ & $1 \mathrm{~s}^{2} 4 \mathrm{p}$ & ${ }^{2} \mathrm{P} 1 / 2$ & $100 \%$ \\
\hline $2.608 \times 10^{-12}$ & $3,764,548$ & $1 \mathrm{~s}^{2} 4 \mathrm{p}$ & ${ }^{2} \mathrm{P} 3 / 2$ & $100 \%$ \\
\hline $2.547 \times 10^{-12}$ & $* 3,774,240$ & $1 \mathrm{~s}^{2} 4 \mathrm{f}$ & ${ }^{2} \mathrm{~F} 5 / 2$ & $100 \%$ \\
\hline $2.553 \times 10^{-12}$ & ${ }^{*} 3,774,380$ & $1 \mathrm{~s}^{2} 4 \mathrm{f}$ & ${ }^{2} \mathrm{~F} 7 / 2$ & $100 \%$ \\
\hline $4.396 \times 10^{-12}$ & ${ }^{*} 4,193,330$ & $1 s^{2} 5 p$ & ${ }^{2} \mathrm{P} 1 / 2$ & $100 \%$ \\
\hline $4.392 \times 10^{-12}$ & ${ }^{*} 4,193,980$ & $1 \mathrm{~s}^{2} 5 \mathrm{p}$ & ${ }^{2} \mathrm{P} 3 / 2$ & $100 \%$ \\
\hline $4.773 \times 10^{-12}$ & $* 4,199,015$ & $1 \mathrm{~s}^{2} 5 \mathrm{f}$ & ${ }^{2} \mathrm{~F} 5 / 2$ & $100 \%$ \\
\hline $4.783 \times 10^{-12}$ & $* 4,199,115$ & $1 \mathrm{~s}^{2} 5 \mathrm{f}$ & ${ }^{2} \mathrm{~F} 7 / 2$ & $100 \%$ \\
\hline $7.060 \times 10^{-12}$ & ${ }^{*} 4,422,548$ & $1 \mathrm{~s}^{2} 6 \mathrm{p}$ & ${ }^{2} \mathrm{P} 1 / 2$ & $100 \%$ \\
\hline $7.055 \times 10^{-12}$ & ${ }^{*} 4,422,920$ & $1 s^{2} 6 p$ & ${ }^{2} \mathrm{P} 3 / 2$ & $100 \%$ \\
\hline $8.124 \times 10^{-12}$ & $* 4,425,825$ & $1 \mathrm{~s}^{2} 6 \mathrm{f}$ & ${ }^{2} \mathrm{~F} 5 / 2$ & $100 \%$ \\
\hline $8.135 \times 10^{-12}$ & $* 4,425,885$ & $1 \mathrm{~s}^{2} 6 \mathrm{f}$ & ${ }^{2} \mathrm{~F} 7 / 2$ & $100 \%$ \\
\hline $2.126 \times 10^{-11}$ & ${ }^{*} 4,426,025$ & $1 \mathrm{~s}^{2} 6 \mathrm{~h}$ & ${ }^{2} \mathrm{H} 9 / 2$ & $100 \%$ \\
\hline $2.127 \times 10^{-11}$ & ${ }^{*} 4,426,050$ & $1 \mathrm{~s}^{2} 6 \mathrm{~h}$ & ${ }^{2} \mathrm{H} 11 / 2$ & $100 \%$ \\
\hline $1.071 \times 10^{-11}$ & ${ }^{*} 4,560,525$ & $1 \mathrm{~s}^{2} 7 \mathrm{p}$ & ${ }^{2} \mathrm{P} 1 / 2$ & $100 \%$ \\
\hline $1.070 \times 10^{-11}$ & $* 4,560,760$ & $1 \mathrm{~s}^{2} 7 \mathrm{p}$ & ${ }^{2} \mathrm{P} 3 / 2$ & $100 \%$ \\
\hline $1.554 \times 10^{-11}$ & $* 4,649,965$ & $1 \mathrm{~s}^{2} 8 \mathrm{p}$ & ${ }^{2} \mathrm{P} 1 / 2$ & $100 \%$ \\
\hline $1.554 \times 10^{-11}$ & $* 4,650,120$ & $1 s^{2} 8 p$ & ${ }^{2} \mathrm{P} 3 / 2$ & $100 \%$ \\
\hline $6.017 \times 10^{-11}$ & $17,024,200$ & $1 \mathrm{~s} 2 \mathrm{~s} 2 \mathrm{p}$ & $\left({ }^{3} \mathrm{~S}\right)^{4} \mathrm{P} 1 / 2$ & $100 \%$ \\
\hline $2.245 \times 10^{-11}$ & $17,028,110$ & $1 \mathrm{~s} 2 \mathrm{~s} 2 \mathrm{p}$ & $\left(\mathrm{S}^{3}\right)^{4} \mathrm{P} 3 / 2$ & $100 \%$ \\
\hline $4.400 \times 10^{-3}$ & ${ }^{*} 17,035,000$ & $1 \mathrm{~s} 2 \mathrm{~s} 2 \mathrm{p}$ & $\left(\mathrm{S}^{3}\right)^{4} \mathrm{P} 5 / 2$ & $100 \%$ \\
\hline $2.219 \times 10^{-14}$ & $17,186,895$ & $1 \mathrm{~s} 2 \mathrm{~s} 2 \mathrm{p}$ & $\left({ }^{1} \mathrm{P}\right)^{2} \mathrm{P} 1 / 2$ & $61 \%+39 \% 1 \mathrm{~s} 2 \mathrm{~s} 2 \mathrm{p}\left({ }^{3} \mathrm{~S}\right)^{2} \mathrm{P}$ \\
\hline $2.067 \times 10^{-14}$ & $17,191,815$ & $1 \mathrm{~s} 2 \mathrm{~s} 2 \mathrm{p}$ & $\left({ }^{3}\right)^{2} P_{3} / 2$ & $50 \%+50 \% 1 \mathrm{~s} 2 \mathrm{~s} 2 \mathrm{p}(\mathrm{S})^{1} \mathrm{P}$ \\
\hline $1.461 \times 10^{-13}$ & $17,261,115$ & $1 \mathrm{~s} 2 \mathrm{~s} 2 \mathrm{p}$ & $\left(^{3} \mathrm{~S}\right)^{2} \mathrm{P} 1 / 2$ & $61 \%+39 \% 1 \mathrm{~s} 2 \mathrm{~s} 2 \mathrm{p}(\mathrm{S}))^{2} \mathrm{P}$ \\
\hline $2.860 \times 10^{-13}$ & $17,264,350$ & $1 \mathrm{~s} 2 \mathrm{~s} 2 \mathrm{p}$ & $\left({ }^{1} \mathrm{P}\right)^{2} \mathrm{P} 3 / 2$ & $50 \%+50 \% 1 \mathrm{~s} 2 \mathrm{~s} 2 \mathrm{p}\left({ }^{3} \mathrm{~S}\right)^{2} \mathrm{P}$ \\
\hline $6.847 \times 10^{-11}$ & $19,920,720$ & $1 \mathrm{~s} 2 \mathrm{~s} 3 \mathrm{p}$ & $\left({ }^{3} \mathrm{~S}\right)^{4} \mathrm{P} 1 / 2$ & $100 \%$ \\
\hline $2.375 \times 10^{-11}$ & $19,921,845$ & $1 \mathrm{~s} 2 \mathrm{~s} 3 \mathrm{p}$ & $\left({ }^{3} \mathrm{~S}\right)^{4} \mathrm{P} 3 / 2$ & $100 \%$ \\
\hline $3.585 \times 10^{-9}$ & ${ }^{*} 19,923,960$ & $1 \mathrm{~s} 2 \mathrm{~s} 3 \mathrm{p}$ & $\left(\mathrm{S}^{3}\right)^{4} \mathrm{P} 5 / 2$ & $100 \%$ \\
\hline $9.801 \times 10^{-14}$ & $19,949,895$ & $1 \mathrm{~s} 2 \mathrm{~s} 3 \mathrm{p}$ & $\left(\mathrm{S}^{3}\right)^{2} \mathrm{P} 3 / 2$ & $99 \%$ \\
\hline $9.787 \times 10^{-14}$ & $19,951,015$ & $1 \mathrm{~s} 2 \mathrm{~s} 3 \mathrm{p}$ & $\left(\mathrm{S}^{3}\right)^{2} \mathrm{P} 1 / 2$ & $100 \%$ \\
\hline $2.294 \times 10^{-13}$ & ${ }^{*} 20,054,080$ & $1 \mathrm{~s} 2 \mathrm{~s} 3 \mathrm{p}$ & $\left({ }^{1} \mathrm{P}\right)^{2} \mathrm{P} 1 / 2$ & $100 \%$ \\
\hline
\end{tabular}




\section{Continued}

$\begin{array}{lllll}2.300 \times 10^{-13} & { }^{*} 20,057,670 & 1 \mathrm{~s} 2 \mathrm{~s} 3 \mathrm{p} & \left({ }^{1} \mathrm{P}\right)^{2} \mathrm{P} 3 / 2 & 100 \% \\ 1.247 \times 10^{-9} & { }^{*} 20,851,270 & 1 \mathrm{~s} 2 \mathrm{~s} 4 \mathrm{p} & \left({ }^{3} \mathrm{~S}\right){ }^{4} \mathrm{P} 1 / 2 & 100 \% \\ 4.749 \times 10^{-10} & { }^{*} 20,851,760 & 1 \mathrm{~s} 2 \mathrm{~s} 4 \mathrm{p} & (\mathrm{S}))^{4} \mathrm{P} 3 / 2 & 100 \% \\ 1.054 \times 10^{-7} & { }^{*} 20,852,600 & 1 \mathrm{~s} 2 \mathrm{~s} 4 \mathrm{p} & \left({ }^{3} \mathrm{~S}\right)^{4} \mathrm{P} 5 / 2 & 100 \% \\ 2.236 \times 10^{-13} & 20,886,130 & 1 \mathrm{~s} 2 \mathrm{~s} 4 \mathrm{p} & \left({ }^{3} \mathrm{~S}\right)^{2} \mathrm{P} 3 / 2 & 99 \% \\ 2.236 \times 10^{-13} & 20,886,620 & 1 \mathrm{~s} 2 \mathrm{~s} 4 \mathrm{p} & (\mathrm{S})^{2} \mathrm{P} 1 / 2 & 99 \% \\ 3.982 \times 10^{-13} & { }^{*} 20,987,940 & 1 \mathrm{~s} 2 \mathrm{~s} 4 \mathrm{p} & \left({ }^{1} \mathrm{P}\right)^{2} \mathrm{P} 1 / 2 & 99 \% \\ 3.982 \times 10^{-13} & * 20,989,430 & 1 \mathrm{~s} 2 \mathrm{~s} 4 \mathrm{p} & \left({ }^{1} \mathrm{P}\right)^{2} \mathrm{P} 3 / 2 & 99 \%\end{array}$

(*) Indicates an attempt to identify.

spectra, as also phosphorus is an astrophysically important element. The present work is part of an ongoing program, whose goal is to obtain weighted oscillator strength, $g f$, and lifetimes for elements of astrophysical importance. Phosphorus occupies the fifteenth place with respect to cosmic distribution [26].

\section{References}

[1] Moore, C.E. (1949) Atomic Energy Levels. Vol. 1, Circular of the National Bureau of Standards, Washington DC.

[2] Kelly, R.L. and Palumbo, L.J. (1973) Atomic and Ionic Emission Lines below 2000 Angstroms: Hydrogen through Krypton. NRL Report 7599, Tune.

[3] Fawcett, B.C. (1970) Classification of Highly Ionized Emission Lines due to Transitions from Singly and Doubly Excited Levels in Sodium, Magnesium, Alumninum, Silicon, Phosphorus, Sulphur, and Chlorine. Journal of Physics B, 3, 1152-1163. http://dx.doi.org/10.1088/0022-3700/3/8/017

[4] Fawcett, B.C., Hardcastle, R.A. and Tondello, G. (1970) New Classifications of Emission Lines of Highly Ionized Phosphorus and Sulphur. Journal of Physics B, 3, 564-571. http://dx.doi.org/10.1088/0022-3700/3/4/011

[5] Goldsmith, S., Oren, L. and Cohen, L. (1973) Spectra of P XII and P XIII in the Extreme Vacuum Ultraviolet. Journal of the Optical Society of America, 63, 352-358. http://dx.doi.org/10.1364/JOSA.63.000352

[6] Kasyanov, Y.S., Konomov, E.Y.A., Korobkin, V.V., Koshelev, K.N. and Serov, R.V. (1973) Intrashell Transitions in the Spectra Multicharged Phosphorus Ions. Optics and Spectroscopy (URSS), 35, 586-589.

[7] Dere, K.P. (1978) Spectral Lines Observed in Solar Flares between 171 and 630 Angstroms. Astrophysics, 221, 10621067.

[8] Deschepper, P., Lebrunm, P., Palffy, L. and Pellegrin, P. (1982) Energy and Lifetime Measurements in Heliumlike and Lithiumlike Phosphorus. Physical Review A, 26, 1271-1277. http://dx.doi.org/10.1103/PhysRevA.26.1271

[9] Edlen, B. (1983) Comparison of Theoretical and Experimental Level Values if the $\mathrm{n}=2$ Complex in Ions Isoelectronic with Li, Be, O and F. Physica Scripta, 28, 51-67. http://dx.doi.org/10.1088/0031-8949/28/1/007

[10] Fawcett, B.C. and Ridgeley, A. (1981) Analysis of $n=3$ to $n=4$ Spectra for Ions from Mg X to Fe XXIV and P XI, XII. Journal of Physics B: Atomic and Molecular Physics, 14, 203-208. http://dx.doi.org/10.1088/0022-3700/14/2/005

[11] Aglitskii, E.V., Boiko, V.A., Zakharov, S.M., Pikuz, S.A. and Faenov, A.Y.A. (1974) Observation in Laser Plasmas and Identification of Dielectron Satellites of Spectral Lines of Hydrogen- and Helium-Like Ions of Elements in the NaV Range. Soviet Journal of Quantum Electronics, 4, 500-513. http://dx.doi.org/10.1070/qe1974v004n04abeh006795

[12] Boiko, V.A., Faenov, A.Y. and Pikuz, S.A. (1978) X-Ray Spectroscopy of Multiply-Charged Ions from Laser Plasmas. Journal of Quantitative Spectroscopy \& Radiative Transfer, 19, 11-50. http://dx.doi.org/10.1016/0022-4073(78)90038-9

[13] Vainshtein, L.A. and Safronova, U. (1975) Wavelengths and Transition Probabilities for Ions. I ISAN Report, Troitsk, Russia, N6, 1-68.

[14] Vainshtein, L.A. and Safronova, U. (1978) Wavelengths and Transition Probabilities of Satellites to Resonance Lines of H- and He-Like Ions. Atomic Data and Nuclear Data Tables, 21, 49-68. http://dx.doi.org/10.1016/0092-640X(78)90003-7

[15] (1980) Wavelengths and Transition Probabilities for Atoms and Atomic Ions_-Part 1: Wavelengths; Part 2: Transition Probabilities. NSRDS-NBS, Washington DC, 68. 
[16] Reader, J. and Corlis, C.H. (1982) Line Spectra of the Elements. In: Weast, R.C., Ed., CRC Handbook of Chemistry and Physics, 63rd Edition, CRC Press, Boca Raton.

[17] Martin, W.C., Zalubas, R. and Musgrove, A. (1985) Energy Levels of Phosphorus, P I through P XV. Journal of Physical and Chemical Reference Data, 14, 796-797. http://dx.doi.org/10.1063/1.555736

[18] Hayes, R.W. and Fawcett, B.C. (1986) The Spectrum of Phosphorus VI to XIII Between 22 and 92 A. Physica Scripta, 34, 337-341. http://dx.doi.org/10.1088/0031-8949/34/4/009

[19] Kelly, R.L. (1987) Atomic and Ionic Spectrum Lines below 2000 Angstroms: Hydrogen through Krypton. Journal of Physical and Chemical Reference Data, 16, 1-1698.

[20] Hu, M.-H. and Wang, Z.-W. (2004) Oscillator Strengths for $2^{2} \mathrm{P}-n^{2} \mathrm{D}$ Transitions of Lithium-Like Systems with $Z=$ 11 to 20. Chinese Physics, 13, 1246-1250. http://dx.doi.org/10.1088/1009-1963/13/8/011

[21] Chen, C. and Wang, Z.-W. (2005) Oscillator Strengths for $2 s^{2}-2 p^{2} P$ Transitions of the Lithium Isoelectronic Sequence from NaIX to CaXVIII. Communications in Theoretical Physics, 43, 305-308. http://dx.doi.org/10.1088/0253-6102/43/2/021

[22] Hu, M.-H. and Wang, Z.-W. (2009) Oscillator Strengths for $2^{2} S-n^{2} P$ Transitions of the Lithium Isoelectronic Sequence from $Z=11$ to 20. Chinese Physics B, 18, 2244-2249. http://dx.doi.org/10.1088/1674-1056/18/6/023

[23] Cowan, R.D. (1981) The Theory of Atomic Structure and Spectra. University of California Press, Berkeley.

[24] Owens, J.C. (1967) Optical Refractive Index of Air: Dependence on Pressure, Temperature and Composition. Applied Optics, 6, 51-59. http://dx.doi.org/10.1364/AO.6.000051

[25] Fawcett, B.C. (1991) On the Accuracy of Oscillator Strengths. In: Wilson, S., Grant, I.P. and Gyorffy, B.L., Eds., The Effects of Relativity in Atoms, Molecules, and the Solid State, Springer, New York, 45-54.

[26] Aller, L.H. (1963) Distribution of the Chemical Elements. Foreign Literature Press, Moscow. 